\title{
In vitro Activity of Sorghum bicolor Extracts, 3-deoxyanthocyanidins, Against
}

\section{Toxoplasma gondii}

\author{
Daniel A. Abugri ${ }^{a, c}$ William H. Witola ${ }^{b, *}$, Jesse M. Jaynes ${ }^{c}$, Nashar Toufic ${ }^{d}$ \\ ${ }^{\mathrm{a}}$ Department of Chemistry, College of Arts and Sciences, Tuskegee University, Tuskegee, \\ Alabama, USA. ${ }^{b}$ Department of Pathobiology, College of Veterinary Medicine, University of \\ Illinois, Urbana-Champaign, USA, ${ }^{\mathrm{c} D e p a r t m e n t ~ o f ~ A g r i c u l t u r a l ~ a n d ~ E n v i r o n m e n t a l ~ S c i e n c e s, ~}$ \\ College of Agriculture, Environment and Nutrition Sciences, ${ }^{\mathrm{d}}$ Department of Pathobiology, \\ College of Veterinary Medicine, Nursing \& Allied Health, Tuskegee University, Tuskegee, \\ Alabama, USA.
}

*Corresponding Author: William H. Witola, Ph.D.

Department of Pathobiology, College of Veterinary Medicine, University of Illinois, UrbanaChampaign, 2001 South Lincoln Avenue, Urbana, IL 61802, USA

Email:whwit35@illinois.edu

Phone: 217-300-3439

Fax: +217-244-7421 


\begin{abstract}
We investigated dried red leaf extracts of $S$. bicolor for activity against Toxoplasma gondii tachyzoites. S. bicolor red leaf extracts were obtained by bioassay-guided fractionation using ethanol and ethyl acetate as solvents. Analysis of the crude and fractionated extracts from $S$. bicolor using electrospray ionization mass spectrometry (ESI-MS) showed that they contained significant amounts of apigeninidin, luteolinidin, 7-methoxyapigeninidin, 5methoxyapigeninidin, 5-methoxyluteolinidin, 5-7-dimethoxyapigeninidin $\quad$ or $\quad 5-7$ dimethoxyluteolinidin, based on mass per charge $(\mathrm{m} / \mathrm{z})$. When tested in vitro, the $\mathrm{IC}_{50 \mathrm{~s}}$ for inhibitory activity against $T$. gondii tachyzoites' growth of the ethanol and ethyl acetate extracts were 2.3- and 4-fold, respectively, lower than their cytotoxic $\mathrm{IC}_{50 \mathrm{~s}}$ in mammalian cells. Ethyl acetate extracts fractionated in chloroform-methanol and chloroform had $\mathrm{IC}_{50 \text { s }}$ against $T$. gondii that were 56.1- and 3-fold lower than their respective cytotoxic $\mathrm{IC}_{50 \mathrm{~s}}$ in mammalian cells. These antiparasitic activities were found to be consistent with those of the respective pure 3deoxyanthocyanidin compounds identified to be contained in the fractions in significant amounts. Further, we observed that, the position and number of methoxy groups possessed by the 3-deoyanthocyanidins influenced their antiparasitic activity. Together, our findings indicate that S. bicolor red-leaf 3-deoxyanthocyanidins-rich extracts have potent in vitro inhibitory activity against the proliferative stage of $T$. gondii parasites.
\end{abstract}

Keywords: Sorghum bicolor; 3-deoxyanthocyanidins extracts; Toxoplasma gondii; antiparasitic compounds 


\section{Introduction}

Toxoplasmosis is a disease caused by Toxoplasma gondii, a zoonotic apicomplexan protozoa of medical and veterinary significance globally. Humans are infected post-natally through the ingestion of undercooked $T$. gondii-infected meat or through ingestion of parasite oocysts in contaminated water or plants (Dubey, 2009). Because of the multiple paths $T$. gondii uses to infect, one in three people globally are infected (Sullivan and Jeffers, 2012). Prevalence of $T$. gondii seropositive humans is markedly high in countries where raw or undercooked meat is consumed. Congenital infections are frequent and cause gross detriment to the health of infected children (Miller et al., 2009).

Several therapeutic agents are available for managing toxoplasmosis in humans, including azithromycin, roxithromycin, spiramycin, pyrimethamine, clindamycin, erythromycin and a combination of pyrimethamine-sulfadiazine (Tee et al., 1998; Chamberland et al., 1991; Alder et al., 1994; Van der Ven et al., 1996). However, these drugs have serious toxic, hypersensitivity and teratogenic side-effects and are not effective against the encysted brachyzoite stage of the parasite (McLead et al., 2006; Fomovska et al., 2012). Thus, there is urgent need to develop a new generation of safe and efficacious drugs for use in controlling T. gondii infections.

Plant extracts containing phenolic compounds and their derivatives, especially, flavonoids, tannins, flavonol, flavone, luteolin, quercetin have been shown to have growth inhibitory activity against apicomplexan protozoan parasites including T. gondii (Weiss et al 1998; Dobbin et al., 2002), Plasmodium falciparum (Lehane and Saliba, 2008), Trypanosoma 
(Mamani-Matsuda et al., 2004), and Leishmania (Kirmizibekmez et al., 2004; Sen et al., 2005). S. bicolor contains abundant amounts of 3-deoxyanthocyanidins compounds (Oladiji et al., 2007). However, there is no available information regarding these compounds' ability to both inhibit and kill $T$. gondii tachyzoites. Thus, in this study we endeavored to extract, fractionate and identify compounds from $S$. bicolor red leaf with activity against $T$. gondii using in vitro bio-assays.

\section{Materials and Methods}

\subsection{Plant material}

Leaves of mature S. bicolor Red plants with grain that is ready for harvest were obtained from Northern Ghana, West Africa. The leaves were air-dried under mild sunlight and then packaged in amber-colored zip-lock bags until use. They were authentically identified as $S$. bicolor Red leaves. A voucher specimen of the leaves has been deposited in the Department of Chemistry, Tuskegee University, Alabama, USA.

\subsection{General analytical and fractionation procedures}

All solvents were of high performance liquid chromatography grade (HPLC grade) obtained from Sigma and Fisher Scientific, USA. The S. bicolor leaf extracts were obtained using procedures reported by Wang et al. (2013) and Abugri et al. (2013). Approximately $41.04 \mathrm{~g}$ of the dried and ground plant samples were mixed with $500 \mathrm{~mL}$ ethanol (99.9\%) and incubated in a water bath with shaking at $100 \mathrm{rpm}$ for $2 \mathrm{~h}$ at $30^{\circ} \mathrm{C}$. The procedure was repeated three more times and the resultant extracted volumes pooled, filtered using Whatman number 2 filter paper and evaporated to dryness under reduced pressure at $60^{\circ} \mathrm{C}$. The obtained crude extract was 
washed with water, followed by successive treatment with n-hexane, ethyl acetate and n-butanol (water saturated) (Wang et al., 2013).

\subsection{Fractionation and purification of compounds from crude ethyl acetate extracts}

Size exclusion chromatography was carried out with Biogel-P60 (BioRad. Inc., USA) on ethyl acetate extracts using solvents of different polarities, namely pure chloroform, chloroformmethanol mixture (10:1), pure methanol or pure ethyl acetate to extract bioactive compounds (Figure 1). The various fractions were collected in $15 \mathrm{~mL}$ Pyrex culture test tubes, dried and analyzed by qualitative thin layer chromatography (TLC) on pre-coated plates (silica gel 60 $\mathrm{F}_{254 \mathrm{~nm}}, 0.2 \mathrm{~mm}$ : Merck). Spots on the plates were visualized under UV light (254) and excised from the plates. The excised spots were eluted from silica gel into their respective solvents and analyzed using Electrospray Ionization-Mass spectrometry (ESI-MS) and High Performance Liquid Chromatography. Identified fractions that were obtained in significant amounts were reconstituted in dimethyl sulphoxide (DMSO) and tested in vitro for growth inhibitory activity against Toxoplasma gondii and for cytotoxicity against parasite host human foreskin fibroblasts as well as human aortic endothelial cells.

\subsection{Characterization of fractionated compounds}

Samples were analyzed using an Ultra Performance LC System (ACQUITY, Waters Corp., Milford, MA, USA) coupled with a quadrupole time-of-flight mass spectrometer (Q-TOF Premier, Waters) with lectrospray ionization (ESI) in negative ESI-MS operated by Masslynx software (V4.1). Each sample, in acetonitrile, was directly injected into the ESI source at a flow rate of $50 \mu \mathrm{L} / \mathrm{min}$ with mobile phase of $100 \%$ or $50 \%$ acetonitrile $(\mathrm{ACN})$ in water. The ion source voltages were set at $3 \mathrm{kV}$ for positive and negative ion mode acquisitions. The sampling cone was set at $37 \mathrm{~V}$ and the extraction cone was at $3 \mathrm{~V}$. In both negative and positive modes, the 
source and desolvation temperatures were maintained at $120^{\circ} \mathrm{C}$ and $225^{\circ} \mathrm{C}$, respectively, with the desolvation gas flow at $200 \mathrm{~L} / \mathrm{h}$. The TOF MS scanning was from 50 to $1500 \mathrm{~m} / \mathrm{z}$ at $1 \mathrm{~s}$ with $0.1 \mathrm{~s}$ inter-scan delay using extended dynamic range acquisition with centriod data format. For real time mass calibration, direct infusion of sodium formate solution $(10 \%$ formic Acid $/ 0.1 \mathrm{M}$ $\mathrm{NaOH} / \mathrm{ACN}$ at a ratio of $1: 1: 8)$ at $1 \mathrm{sec} / 10 \mathrm{sec}$ to ion source at $2 \mu \mathrm{l} / \mathrm{min}$ was used for a single point mass calibration.

Ions of interest were analyzed for elemental composition using accurate mass (less than $5 \mathrm{ppm}$ error) and isotope modeling to identify the chemical formulae. Collision-induced dissociation (CID) by argon on precursor ions resulted in structural fragmentation that further assisted the identification.

\subsection{Maintenance of Parasites and Cell Culture}

Confluent monolayers of human foreskin fibroblasts (HFF) and aortic endothelial cells (HAEC) were maintained in Iscove's modified Dulbecco's medium (IMDM) supplemented with 10\% (v/v) heat-inactivated fetal bovine serum (Life Technologies), 1\% (v/v) Glutamax and 1\% (v/v) penicillin-streptomycin-fungizone (Life Technologies) at $37^{\circ} \mathrm{C}$ with $5 \% \mathrm{CO}_{2}$. Toxoplasma gondii RH strain parasites, constitutively expressing cytosolic yellow fluorescent protein (RH-YFP) (Gubbels et al., 2003) were maintained in HFF cells. For parasite growth inhibitory activity tests of the compounds, HFF cells were seeded into 96 well plates with a total volume of $200 \mu \mathrm{L}$ of medium and allowed to proliferate to full confluency prior to use. 


\subsection{Preparation of compounds for toxicity Assays.}

The dried initial crude ethanol extract, the ethyl acetate extract and fractionated compounds, as well as pure synthetic compounds, were reconstituted in dimethyl sulphoxide (DMSO). When compounds were added to cell cultures, the final concentration of DMSO did not exceed $1 \%$.

\subsection{Analysis of compounds' in vitro inhibitory activity against T. gondii}

Confluent HFF cells grown in 96 well plates were inoculated with RH-YFP T. gondii tachyzoites at a concentration of 3,500 parasites $/ \mathrm{ml}$. Test compounds at increasing concentrations were added immediately after inoculation in triplicate. Cultures without compounds (but with similar volume of DMSO) were maintained as controls. At 24, 48 and $72 \mathrm{~h}$ post-infection, parasite proliferation was determined using an image Xpress micro automated fluorescence microscope by measuring the parasite YFP fluorescence followed by quantification of YFP intensity using ImageJ version $1.37 \mathrm{v}$ software $(\mathrm{NIH})$. The $\mathrm{IC}_{50}$ of the compounds against $T$. gondii were determined using nonlinear regression analysis by Graph pad Prism software version 6.0 program (CA, USA).

\subsection{Cytotoxicity in HFF cells}

HFF cells were seeded into 12 well plates at a concentration of $10^{5}$ cells $/ \mathrm{ml}$ and a total volume of $1 \mathrm{~mL}$ medium per well. The cells were maintained until $100 \%$ confluency after which $1 \mathrm{~mL}$ of the old medium was aspirated out and replaced with an equal volume of fresh medium. Different concentrations of crude ethanol extract or fractionated or pure compounds were added to culture wells in triplicate for a total of three independent experiments. DMSO was used as a negative control. After $48 \mathrm{~h}$ of incubation at $37^{\circ} \mathrm{C}$ with an atmosphere of $5 \% \mathrm{CO}_{2}$, the medium was aspirated out from the wells and $250 \mu \mathrm{L} 1 \mathrm{x}$ trypsin (Gibco) added followed by 2 minute 
incubation at room temperature. Then, $1 \mathrm{~mL}$ of fresh medium containing $10 \% \mathrm{FBS}$ was added and mixed by pipetting gently. Exactly $20 \mu \mathrm{L}$ of the cell suspension was transferred to a $1.5 \mathrm{~mL}$ microcentrifuge tube and an equal volume of trypan blue (BioRad) added and mixed gently. Exactly $10 \mu \mathrm{L}$ of the mixture was pipetted into a counting slide chamber and the number of live and dead cells counted using a TCA 20 automated cell counter (BioRad).

\subsection{Cell viability assays}

Confluent HFF and HAEC cells maintained in supplemented IMDM medium (without red phenol) in 96-well plates were used. Prior to addition of the compounds, $150 \mu \mathrm{L}$ of medium was aspirated out of each well and replaced with an equal volume of fresh medium. Varying concentrations of the ethanol extract, ethyl acetate extract and the two ethyl acetate fractions were added to the wells in triplicate. Triplicate wells to which an equivalent volume of DMSO only was added were included as negative control. At 48 hours of culture, a colorimetric assay using the cell proliferation reagent WST-1 (Roche) for the quantification of cell viability was performed on the cultures by adding $20 \mu \mathrm{L}$ of the WST-1 reagent to each well. After mixing, the plates were wrapped in aluminum foil and incubated for $1 \mathrm{~h}$ at $37^{\circ} \mathrm{C}$ with $5 \% \mathrm{CO}_{2}$. After 1 hour of incubation, exactly $150 \mu \mathrm{L}$ of the medium from each well was transferred to a new 96-well plate and quantification of the formazan dye produced by metabolically active cells was read as absorbance at a wavelength of $420 \mathrm{~nm}$ using a scanning multi-well spectrophotometer (Spectra Max 250; Molecular Devices). Three independent assays were performed and the dose-response curves of the means of triplicate assays for each compound were generated using GraphPad PRISM software and the $\mathrm{IC}_{50}$ values derived. 


\subsection{Statistical analysis}

The selectivity index (SI) was determined as the ratio of minimum cytotoxic concentration at $50 \%\left(\mathrm{IC}_{50 \mathrm{c}}\right)$ to minimum parasite growth inhibitory concentration at 50\% $\left(\mathrm{IC}_{50} \mathrm{p}\right)$. The statistical difference of effect of test compounds and fractions for the inhibition of $T$. gondii replication and survival were compared with control group or compared between different extracts, fractions and pure compounds using Welch's t-test on non-homogeneous variances $(p<0.05)$. The dosedependent effect of inhibitory activities against $T$. gondii of the crude extracts, fractions, apigeninidin and luteolinidin were determined by non-linear regression. The dose-response curves were derived using GraphPad PRISM software. Calculation of confidence intervals was done at the level of $95 \%$. All data are reported as triplicate assays. Analyses of the differences between the compounds' effective $\mathrm{IC}_{50}$ against the parasite compared to the cytotoxic $\mathrm{IC}_{50}$ in mammalian cells were done using two-tailed Student's $t$ test $(p<0.05)$.

\subsection{Results}

\subsection{Fractionation and identification of Compounds}

The anti-Toxoplasma activity of crude ethanol extracts, ethyl acetate, ESI-MS-identified ethyl acetate-fractionated compounds from S. bicolor red leaf, as well as that of the synthetic 3deoxyanthocyanidins (apigeninidin, luteolinidin, 7-methoxyapigeninidin, 5, 7dimethoxyluteolinidin), were tested (Table 1). The major 3-deoxyanthocyanidins found in the extracts and fractionated samples of S. bicolor leaf were apigeninidin and luteolinidin, that constituted more than $2 \%$ relative to all other 3-deoxyanthocyanidins derivatives (Figure 2-4). The different fractions analyzed depicted distinct ion counts for the major deoxyanthocyanidins present (Figure 2-4). 


\subsection{Testing of fractions' inhibitory activity against T. gondii and cytotoxicity}

To determine the S. bicolor extract that had potent activity against $T$. gondii, but with less cytotoxicity to mammalian cells, we measured the $\mathrm{IC}_{50 \text { s }}$ of the crude ethanol extract and the crude ethyl acetate extract against $T$. gondii tachyzoites and against human fibroblasts (HFF). The crude ethanol extract had an $\mathrm{HFF}$ cytotoxic $\mathrm{IC}_{50}$ that was 6-fold higher than that of the crude ethyl acetate extract (Table 1). However, the ethyl acetate extract was found to have significant activity against $T$. gondii tachyzoites, with a mean $\mathrm{IC}_{50}$ that was about 15 -fold lower than that of the ethanol crude extract (Table 1), implying that the ethyl acetate extract had more potent activity against $T$. gondii than the ethanol extracts. Thus, the ethyl acetate crude extract was fractionated and the activity of its fractions against $T$. gondii and HFF cells tested. The two ethyl acetate fractions (EA F1-F4 and EA F2-5) had lower (though not significant) HFF cytotoxic $\mathrm{IC}_{50 \text { s, }}$ but were potently active against $T$. gondii tachyzoites with $\mathrm{IC}_{50 \mathrm{~s}}$ comparable to that of the crude extract (Table 1). The data from which the $\mathrm{IC}_{50 \text { s }}$ of the extracts, fractions and pure compounds were derived by nonlinear regression analysis are presented in graphical form in Figure 5 and Figure 6 and depict compound concentration-dependent decline in parasite growth.

Because the ESI-MS analysis of the ethyl acetate fractions indicated that they contained significant amounts of 3-deoxyanthocyanidins, we tested the activity of commercially-obtained pure 3-deoxyanthocyanidins compounds (apigeninidin chloride, luteolinidin chloride, 7methoxyapigeninidin and 5, 7-dimethoxyapigeninidin). Compared to the ethyl acetate fractions of S. bicolor, the pure 3-deoxyanthocyanidins compounds depicted cytotoxic $\mathrm{IC}_{50 \mathrm{~s}}$ that were 3-4fold lower, but had $\mathrm{IC}_{50 \mathrm{~s}}$ for inhibition of $T$. gondii that were similar to those of ethyl acetate 
fractions (Table 1). This implied that the ethyl acetate fractions from S. bicolor were as potent as the pure synthetic 3-deoxyanthocyanidins against $T$. gondii but had much lower cytotoxicity to mammalian cells than the pure synthetic 3-deoxyanthocyanidins compounds. Further, the synthetic 5,7-dimethoxyluteolinidin methoxy groups exhibited the highest inhibition against parasite growth at $48 \mathrm{~h}$ than the apigeninidin and luteolinidin without one or two methoxy groups at $95 \%$ confidence interval (Table 1).

Table 1. T. gondii growth inhibitory and cytotoxic concentrations $\left(\mathrm{IC}_{50}\right)$ for the $S$. bicolor red leaf crude extracts, fractionated compounds and 3-deoxyathocyanidins pure synthetic compounds

\begin{tabular}{lll}
\hline Compound Tested & $\mathrm{IC}_{50 \mathrm{c}}{ }^{*}(\mu \mathrm{g} / \mathrm{mL})$ & $\mathrm{IC}_{50 \mathrm{p} @ 48 \mathrm{hrr}}{ }^{*}(\mu \mathrm{g} / \mathrm{mL})$ \\
\hline Ethanol Extract & $46.67(41.63-52.32)$ & $20.38(17.86-23.24)$ \\
Ethyl acetate Extract & $7.56(4.56-12.53)$ & $1.39(0.87-2.22)$ \\
EA F1-F4 (C/M 10:1) & $20.20(18.72-21.79)$ & $0.36(0.17-0.74)$ \\
EA F2-5 (chloroform) & $20.39(16.85-24.67)$ & $7.00(4.89-10.05)$ \\
Apigeninidin chloride & $5.24(4.73-5.81)$ & $1.08(1.03-1.13)$ \\
Luteolinidin chloride & $6.54(4.79-8.94)$ & $0.66(0.45-0.97)$ \\
7-methoxyapigeninidin & $7.49(5.025-11.18)$ & $1.48(1.24-1.77)$ \\
5,7-dimethoxyluteolinidin & $2.62(1.68-4.08)$ & $0.53(0.18-1.57)$ \\
\hline
\end{tabular}

${ }^{*}$ Results in the table are reported as means of three independent assays at $95 \%$ confidence interval; $\mathrm{IC}_{50 \mathrm{c}}$ : minimum inhibitory concentration required to kill $50 \%$ of $\mathrm{HFF}$ cells; $\mathrm{IC}_{50 \mathrm{p}}$-minimum inhibitory concentration of crude, fractionated and pure compounds required to inhibit $T$. gondii tachyzoite growth by $50 \%$ in culture; EA F- ethyl acetate fraction; $\mathrm{C} / \mathrm{M}$ is hexane-methanol.

Table 2. Selective index determination for S. bicolor red leaf crude extracts, fractionated compounds and 3-deoxyathocyanidins pure synthetic compounds

\begin{tabular}{|c|c|}
\hline Compound Tested & $\mathrm{SI}_{@ 48 \mathrm{hrs}}$ \\
\hline Ethanol Extract & 2.29 \\
\hline Ethyl acetate Extract & 5.44 \\
\hline EA F1-F4 (C/M 10:1) & 56.11 \\
\hline EA F2-5 (chloroform) & 2.91 \\
\hline Apigeninidin chloride & 4.85 \\
\hline Luteolinidin chloride & 9.91 \\
\hline 7-methoxyapigeninidin & 5.06 \\
\hline 5,7-dimethoxyluteolinidin & 4.94 \\
\hline
\end{tabular}

Results in the table are reported as means of three independent assays at $95 \%$ confidence interval. EA F- ethyl acetate fraction; $\mathrm{C} / \mathrm{M}$ is hexane-methanol; SI-selectivity index $\left(\mathrm{IC}_{50 \text { cytotoxicity }} / \mathrm{IC}_{50 T}\right.$. gondii inhibition $)$. 
Table 3. Cytotoxic concentrations $\left(\mathrm{IC}_{50}\right)$ for the $S$. bicolor red leaf crude extracts and fractionated compounds in HFF and HAEC cells derived by the in vitro cell viability assay

\begin{tabular}{lll}
\hline Compound & $\mathrm{IC}_{50}(\mu \mathrm{g} / \mathrm{mL})$ & $\mathrm{IC}_{50}(\mu \mathrm{g} / \mathrm{mL})$ \\
& $\mathrm{In} \mathrm{HFF} \mathrm{Cells}$ & $\mathrm{In} \mathrm{HAEC} \mathrm{Cells}$ \\
\hline Ethanol Extract & $40.89 \pm 3.51$ & $39.62 \pm 2.74$ \\
Ethyl acetate Extract & $5.92 \pm 1.63$ & $4.97 \pm 1.31$ \\
EA F1-F4 (C/M 10:1) & $13.74 \pm 2.15$ & $11.82 \pm 1.77$ \\
EA F2-5 (chloroform) & $14.11 \pm 2.36$ & $15.26 \pm 2.46$ \\
\hline
\end{tabular}

Results in the table are reported as means of three independent assays \pm Standard Error of the Mean; HFF: human foreskin fibroblasts; HAEC: human aortic endothelial cells; $\mathrm{IC}_{50}$ : minimum inhibitory concentration required to decrease the metabolic activity of the cells by $50 \%$; EA F- ethyl acetate fraction; $\mathrm{C} / \mathrm{M}$ is hexane-methanol

Apigeninidin and luteolinidin were both found to have significant inhibitory activity against $T$. gondii at $48 \mathrm{~h}$ of culture (Table 1). However, the selectivity index (SI) calculated for luteolinidin was higher than that of apigeninidin (Table 2), indicating that luteolinidin had greater inhibitory activity against $T$. gondii than apigeninidin. The ethyl acetate extract as well as fractions derived from ethyl acetate extract depicted higher SI against $T$. gondii than the ethanol extract (Table 2). Overall, the EA F1-F4 fraction from the ethyl acetate crude extract (fractionated using chloroform-methanol) had the highest SI, which was at least 40-fold higher than that of all other extracts, fractions and pure compounds. All crude and fractionated compounds had cytotoxic $\mathrm{IC}_{50 \mathrm{~s}}$ in human fibroblasts that were at least 2-fold higher than their respective $\mathrm{IC}_{50 \mathrm{~s}}$ against $T$. gondii (Table 1), implying that their effective concentration against the parasite would not have detrimental side-effects in humans. Similarly, the pure, synthetic compounds had cytotoxic $\mathrm{IC}_{50 \text { s }}$ in human fibroblasts that were at least 2-fold higher than their respective $T$. gondii $\mathrm{IC}_{50 \text { s. }}$ However, the levels of concentrations for pure compounds were generally lower than those of the extracts, indicating that high level of purity of the compounds enhanced their activity. The above stated cytotoxic $\mathrm{IC}_{50 \mathrm{~s}}$ of the compounds in HFF cells were derived using an assay based on distinguishing between dead and live cells. Thus, to determine the effect of the compounds on 
the metabolic activity of the mammalian cells, we performed a cell proliferation assay for the quantification of cellular mitochondrial dehydrogenases activity of HFF and HAEC cells. As, shown in Table 3 , cytotoxic $\mathrm{IC}_{50 \text { s }}$ of the compounds based on cellular metabolic activity corroborated those obtained based on cell death (Table 1), indicating that the effective concentrations of the compounds against the parasites were not toxic to mammalian cells (human foreskin fibroblasts and human aortic endothelial cells) tested in this study.

To determine if the number and position of methoxy groups in the 3-deoxyanthocyanidin compounds' B-ring has effect on their inhibitory activity against $T$. gondii, we compared the activity of 7- methoxyapigeninidin and 5, 7-dimethoxyluteolinidin. The activity of 5, 7dimethoxyluteolinidin $\left(\mathrm{IC}_{50}=0.53 \mu \mathrm{g} / \mathrm{mL}\right)$ with two methoxy groups in position C-5 and C-7 was significantly higher than that of 7-methoxyapigeninidin $\left(\mathrm{IC}_{50}=1.48 \mu \mathrm{g} / \mathrm{mL}\right)$ with one methoxy group in position $\mathrm{C}-7$ at $48 \mathrm{~h}$ of parasite culture. Importantly, 7-methoxyapigeninidin had lower cytotoxicity $\left(\mathrm{IC}_{50}=7.49 \mu \mathrm{g} / \mathrm{mL}\right)$ in human fibroblasts than 5,7-dimethoxyluteolinidin $\left(\mathrm{IC}_{50}=2.62 \mu \mathrm{g} / \mathrm{mL}\right)$. The selectivity indexes (SI) for 7- methoxyapigeninidin and 5, 7dimethoxyluteolinidin were 5.06 and 4.94, respectively, at $48 \mathrm{~h}$ of culture (Table 2). This suggested that 7- methoxyapigeninidin possesses higher potential activity against $T$. gondii than 5, 7-dimethoxyluteolinidin.

In comparison to existing drugs, the $\mathrm{IC}_{50 \mathrm{~s}}$ obtained for the ethyl acetate crude extract and its two fractions (EA F1-F4 and EA F2-5) were all notably lower than those of roxithromycin, spiramycin and azithromycin that have $\mathrm{IC}_{50 \mathrm{~s}}$ of $27.07,20.16$ and $8.61 \mu \mathrm{g} / \mathrm{mL}$, respectively (Chamberland et al., 1991). However, we observed that pyrimethamine has been reported to have 
an $\mathrm{IC}_{50}$ of $0.16 \mu \mathrm{g} / \mathrm{mL}$ against $T$. gondii that is lower than the $\mathrm{IC}_{50 \mathrm{~s}}$ for all the extracts we obtained from S. bicolor (Cantin and Chamberland, 1993). A combination of sulfadiazine and pyrimethamine has an $\mathrm{IC}_{50}$ that is significantly lower than the EA F2-5 fraction of the ethyl extract ((Derouin and Chastang, 1989). Nevertheless, it is noteworthy that these existing drugs have been reported to have severe toxic side effects in humans (McLead et al., 2006).

\subsection{Discussion}

Herein, we tested inhibitory activity of extracts from dried $S$. bicolor red leaf against $T$. gondii tachyzoites. The $S$. bicolor crude extracts, fractions and pure compounds were observed to have significant inhibitory activity against $T$. gondii tachyzoites at $\mu \mathrm{g} / \mathrm{ml}$ level. Importantly, their cytotoxic concentrations were at least 2-fold higher than their respective effective concentrations against $T$. gondii, suggesting that they would not have adverse side-effects in vivo. S. bicolor has been shown to contain abundant amounts of 3-deoxyanthocyanidins compounds (Oladiji et al., 2007). Consistent with this report, the results of our ESI-MS chromatographic analysis of $S$. bicolor extracts and fractions revealed that they contained significantly relative high amounts of 3-deoxyanthocyanidins compounds. Importantly, when we tested pure, commercially obtained 3deoxyanthocyanidins compounds, we observed that they had $T$. gondii inhibitory effects that were comparable to the $S$. bicolor extracts and fractions. Taken together, this indicated that the observed activity against $T$. gondii of the fractionated compounds from $S$. bicolor was attributable to the 3-deoxyanthocyanidins that they contained, implying that $S$. bicolor can be a source of bioactive compounds for the development of a new generation of effective and safe drugs against $T$. gondii infections. 
Plant-derived natural products are now increasingly being found to have anti-Toxoplasma activity (Sepulveda-Arias et al., 2014). It is noteworthy that traditionally, in West Africa, $S$. bicolor grains, leaves, roots and stalks are considered to have medicinal properties and are thus used in the treatment of a range of ailments (Oladiji et al., 2007; Abugri et al., 2013). In this study, the 3-deoxyanthocyanidins compounds fractionated from S. bicolor were found to have more potent anti-parasitic activity than other flavonoid compounds reported in previous studies (Lehane and Saliba, 2008). In particular, the $\mathrm{IC}_{50 \mathrm{~s}}$ against $T$. gondii for the ethyl acetate crude extract and its fractions, as well as the pure 3-deoxyanthocyanidins compounds tested in this study, were lower than those reported in different traditional medicinal plants against $T$. gondii tachyzoites (Choi et al., 2008). Additionally, the $\mathrm{IC}_{50 \text { s }}$ against $T$. gondii for the $S$. bicolor extracts and fractions we tested were lower than those of the standard compounds/drugs such as roxithromycin, azithromycin, spiramycin, and pyrimethamine-sulfadiazine used for treating $T$. gondii infections (Derouin et al., 1989; Chamberland et al., 1991; Cantin and Chamberland, 1993).

Our observation that 7-dimethoxyluteolinidin possesses higher potential activity against $T$. gondii than 5,7- methoxyapigeninindin based on the position and number of the methoxy group was consistent with previous studies that showed that synthetic 7-methoxyapigeninidin exhibited higher activity against fungi (G. sorgi) than apigeninidin which lacks methoxy group at position C-7 (Aida et al., 1996).

Even though flavonoids have been shown to possess anti-parasitic activity, most of them have also been shown to be toxic to mammalian cells at their effective concentrations (Matsuo et al., 
2005). Importantly, the S. bicolor extracts and fractions tested in this study had cytotoxic $\mathrm{IC}_{50 \text { s }}$ against human fibroblasts and human endothelial cells that were at least 2-fold higher than their respective $\mathrm{IC}_{50 \text { s }}$ against $T$. gondii. Thus, $S$. bicolor extracts and fractions investigated in this study are promising in that their effective anti-parasitic $\mathrm{IC}_{50 \mathrm{~s}}$ were significantly lower than their respective cytotoxic $\mathrm{IC}_{50 \mathrm{~s}}$. Studies are underway to determine the mechanism of action of 3deoxyanthocyanidins in inhibiting growth and proliferation of $T$. gondii. Once determined, their modes of action will serve as a guide in establishing the combination types and concentrations of 3-deoxyanthocyanidins that would have synergistic or additive effects in order to improve their efficacy and reduce their cytotoxicity, thus making them safer and effective for use in treating $T$. gondii infections in humans.

\section{Acknowledgements}

This study was funded by the USDA/NIFA/ AFRI/ grant 2012-67016-19450 to WHW. We are grateful to the Department of Chemistry and Dr. Wendell McElhenney of Tuskegee University for their support. We extend our sincere gratitudes to the RCMI core Lab and grant number G12MD007585-23 for the support in the microscopy work.

Competing interests: None.

Ethical approval: Not required. 


\section{Figure Legends}

Figure 1: Flow-chart for fractionation of ethanol extracts from Sorghum bicolor using size exclusion chromatography. AcOET: ethyl acetate; MeOH: pure methanol.

Figure 2: ESI-MS chromatogram of compounds extracted from $S$. bicolor. The 3deoxyathocyaninindins compounds were fractionated in chloroform-methanol (10:1 ratio) from the ethyl-acetate crude extract at a stock concentration of $1.398 \mathrm{mg} / \mathrm{ml}$. The compounds, 7methoxy-apidenindin and luteolinidin accounted for $3.04 \%$ and $52.76 \%$, respectively, of the total count ion content in the crude extract.

Figure 3: ESI-MS chromatogram of compounds extracted from $S$. bicolor. The 3deoxyathocyaninidins were fractionated in chloroform from the ethyl-acetate crude extract at a stock concentration of $3.80 \mathrm{mg} / \mathrm{ml}$. Apigeninindin, 7-methoxy-apigenidin, luteolinidin and 7methoxy-luteolinidin accounted for $4.61 \%, 2.75 \%, 36.87 \%$ and $2.50 \%$, respectively, of the total ion count in the crude extract.

Figure 4: ESI-MS chromatogram of compounds extracted from S. bicolor. The 3deoxyathocyaninidins were fractionated in hexane-methanol from the ethyl-acetate crude extract at a stock concentration of $18.389 \mathrm{mg} / \mathrm{mL}$. Apigeninindin, 7-methoxy-apigenidin, luteolinidin and 7-methoxy-luteolinidin accounted for $4.44 \%, 0.72 \%, 1.38 \%$ and $0.57 \%$, respectively, of the total ion count in the crude extract.

Figure 5: Growth curves for $T$.gondii tachyzoites in cultures treated with varying concentrations of compounds. Confluent human foreskin fibroblasts (HFF) were inoculated with T. gondii tachyzoites and immediately treated with varying concentrations $(\mu \mathrm{g} / \mathrm{mL})$ of (A.) Ethanol crude 
extract, (B.) Ethyl acetate crude extract, (C.) Ethyl acetate fraction F2-5 and (D.) Ethyl acetate fraction F1-F4, for 48 hours. The graphs depict compound dose-response patterns of decline in growth. The data are representative of means of three independent assays at $95 \%$ confidence $\operatorname{limit}(95 \% \mathrm{CL})$.

Figure 6: Growth curves for $T$.gondii tachyziotes in cultures treated with varying concentrations of pure compounds. Confluent human foreskin fibroblasts (HFF) were inoculated with $T$. gondii tachyzoites and immediately treated with varying concentrations $(\mu \mathrm{g} / \mathrm{mL})$ of (A.) Luteolinidin, (B.) 7-Methoxyapigeninidin, (C.) 5,7-Dimethoxyluteolinidin and (D.) Apigeninidin, for 48 hours. The graphs depict compound dose-response decline in growth. The data are representative of means of three independent assays at 95\% confidence limit (95\% CL).

\section{References}

Abugri DA, Tiimob JB, Apalagya VA, Pritchett G and McElhenney WH. Bioactive and nutritive compounds in Sorghum bicolor (Guinea corn) red leaves and their health implication. Food Chem. 2013; 138:718-23.

Aida Y, Tamogami S, Kodama O, Tsukiboshi T. Synthesis of 7-methoxyapigeninindin and its fungicidal activity against Gloeocercospora sorghi. Biosci. Biotechnol. Biochem. 1996; 60: 1495-1496

Alder J, Hutch T, Meulbroek JA, Clement JC. Treatment of experimental Toxoplasma gondii infection by clarithromycin-based combination therapy with minocycline or pyrimethamine. J. Acquir. Immune Defic. Syndr. 1994; 7:1141-8. 
Cantin L, Chamberland S. In evaluation of the activities of Azithromycin alone and combined with pyrimethamine against Toxoplasma gondii. 1993:1993-1996.

Chamberland S, Kirst HA, Current WL. Comparative activity of macrolides against Toxoplasma gondii demonstrating utility of an in vitro microassay. Antimicrob. Agents Chemother. 1991; 35: 903-9.

Choi KM, Gang J, Yun J. Anti-Toxoplasma gondii RH strain activity of herbal extracts used in traditional medicine. Int. J. Antimicrob. Agents. 2008; 32: 360-2.

Derouin F and Chastang C. In vitro effects of Folate Inhibitors on Toxoplasma gondii. Antimicrob. Agents Chemother. 1989:1753-1759.

Dobbin CA, Smith NC, Johnson AM. Heat shock protein 70 is a potential virulence factor in murine toxoplasma infection via immunomodulation of host NF-kappa B and nitric oxide. J. Immunol. 2002; 169:958-965.

Dubey JP. Toxoplasmosis in sheep-the last 20 years. Vet. Parasitol. 2009; 163:1-14

Fomovska A, Huang Q, El Bissati K, Mui EJ, Witola WH, Cheng G, Zhou Y, et al. Novel NBenzoyl-2-Hydroxybenzamide disrupts unique parasite secretory pathway. Antimicrob. Agents Chemother. 2012; 56: 2666-2682.

Gubbels MJ, Li C, Striepen B. High-throughput growth assay for Toxoplasma gondii using yellow fluorescent protein. Antimicrob. Agents Chemother. 2003:47: 309-316.

Kirmizibekmez H, Calis I, Perozzo R, Brun R, Donmez AA, Linden A, Ruedi P, Tasdemir D. Inhibiting activities of the secondary metabolites of Phlomis brunneogaleata against parasitic 
protozoa and plasmodial enoyl-ACP Reductase, a crucial enzyme in fatty acid biosynthesis. Planta Med. 2004; 70: 711-717.

Lehane AM, Saliba K. Common dietary flavonoids inhibit the growth of the intraerythrocytic malaria parasite. BMC Research Notes.2008; 1:1-5.

Mamani-Matsuda M, Rambert J, Malvy D, Lejoly-Boisseau H, Daulouede S, Thiolat D, Coves S, Courtois P, Vincendeau P, Mossalayi M.D.Quercetin induces apoptosis of Trypanosoma brucei gambiense and decreases the proinflammatory response of human macrophages. Antimicrob. Agents Chemother. 2004; 48: 924-929.

Matsuo M, Sakaki N, Saga K, Kaneko T. Cytotoxicity of Flavonoids towards cultured normal cells. Biol. Pharm. Bull. 2005; 28:253-259.

McLeod R, Khan AR, Noble GA, Latkany P, Jalbrzikowski J, Boyer K. Toxoplasmosis Study Group. Severe sulfadiazine hypersensitivity in a child with reactivated congenital toxoplasmic chorioretinitis. Pediatr. Infect. Dis. J. 2006; 25: 270-272.

Miller CM, Boulter NR, Ikin RJ, Smith NC. The immunobiology of the innate response to Toxoplasma gondii. Int. J. Parasitol. 2009; 39: 23-39.

Oladiji AT, Jacob TO, Yakubu MT. Anti-anaemic potentials of aqueous extract of Sorghum bicolor (L.) moench stem bark in rats. J. Ethnopharmacol. 2007; 111: 651-6.

Sen G, Mandal S, Roy SS, Mukhopadhyay S, Biswas T. Therapeutic use of quercetin in the control of infection and anemia associated with visceral leishmaniasis. Free Radical Biol. Med. 2005; 38: 1257-1264. 
Sepulveda-Arias JC, Veloza LA, Mantilla-Muriel LE. Anti-Toxoplasma activity of natural products: a review. Recent Pat. Antiinfect. Drug Discov. 2014;9:186-194.

Shih CH, Siu SO, Ng R, Wong E, Chiu LCM, Chu IK, Lo C. Quantitative analysis of anticancer 3-Deoxyanthocyanidins in infected Sorghum seedlings. J. Agric. Food. Chem. 2007; 55: 254259.

Sullivan WJ, Jeffers V. Mechanisms of Toxoplasma gondii persistence and latency. FEMS Microbiology Reviews. 2012; 36:717-733.

Tee GH, Khairul AA, Rohela M, Rahmah N. In vitro evaluation of various drugs against Toxoplasma gondii. Med. J. Malaysia. 1998; 53: 175-180.

Van der Ven AJ, Schoondermark-van de Ven EM, Camps W, Melchers WJ, Koopmans PP, van der Meer JW, Galama JM. Anti-toxoplasma effect of pyrimethamine, trimethoprim and sulphonamides alone and in combination: implications for therapy. J. Antimicrob. Chemother. $1996 ; 38: 75-80$.

Wang Q, Cheng XL, Li H, Quin XY, Ge CY, Liu R, Qi LW, Qin MJ. Application of an efficient strategy for discovery and purification of bioactive compounds from Chinese herbal medicines, a case study on the Puerariae thomsonii Flos. J. Pharm. Biomed. Anal. 2013; 75: 25-32.

Weiss LM, Ma YF, Takvorian PM, Tanowitz HB, Wittner M. Bradyzote development in Toxoplasma gondii and the hsp70 Stress Response. Infect Immun. 1998; 66(7): 3295-3302. 


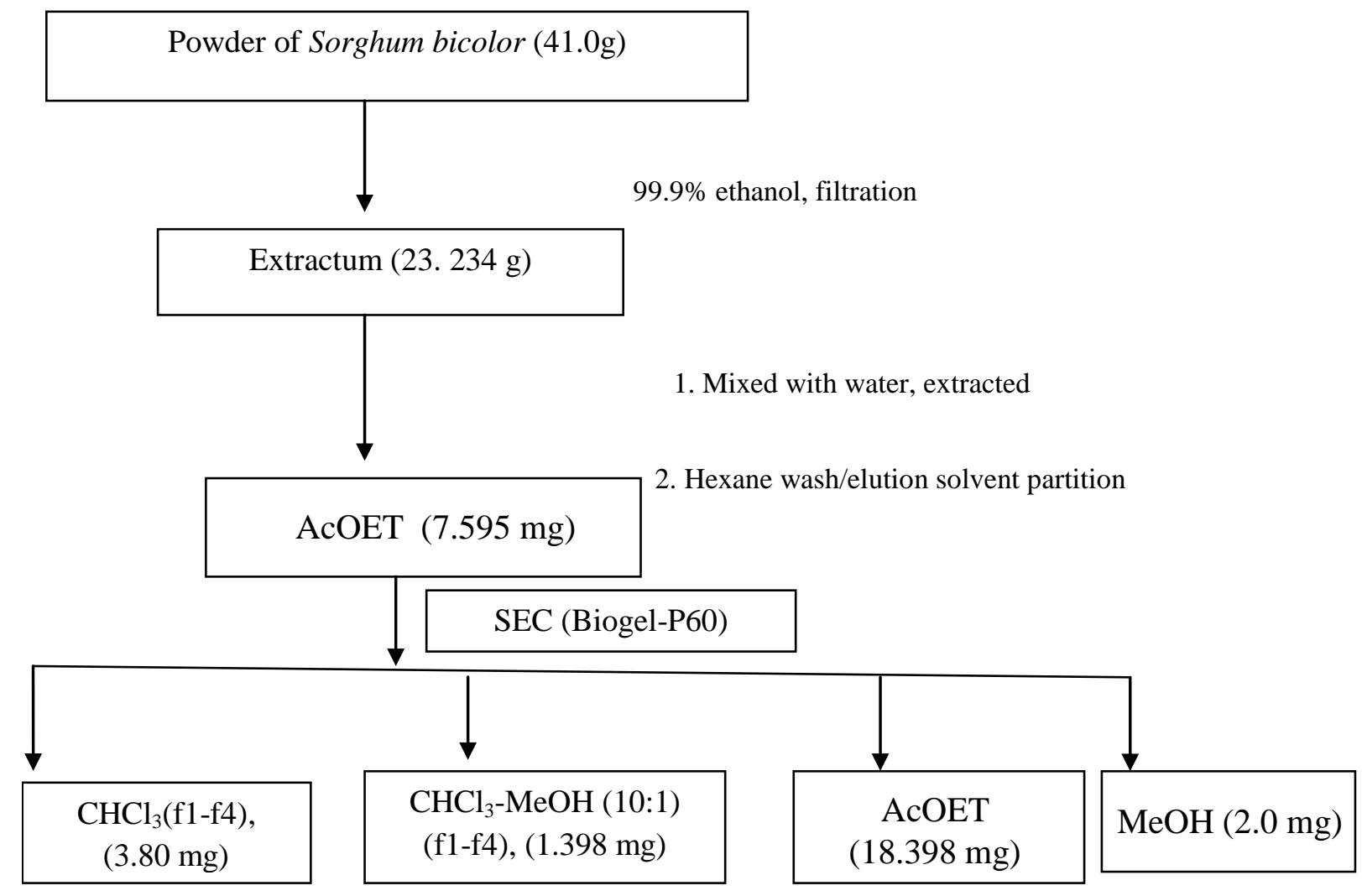

Figure 1: Flow-chart for fractionation of ethanol extracts from Sorghum bicolor using size exclusion chromatography. AcOET: ethyl acetate; $\mathrm{MeOH}$ : pure methanol. 


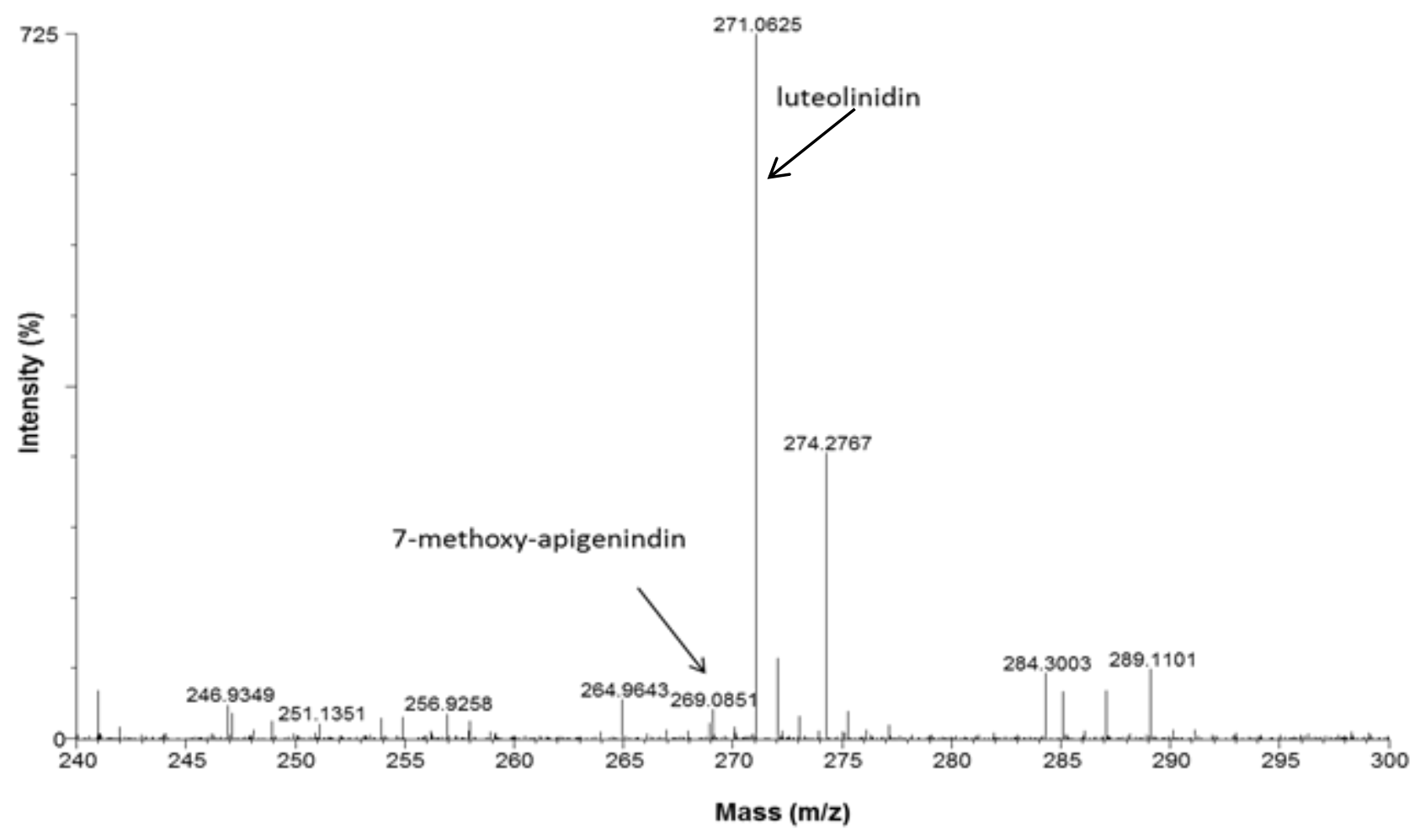

Figure 2. ESI-MS chromatogram of compounds extracted from S. bicolor. The 3-deoxyathocyaninindins compounds were fractionated in chloroform-methanol (10:1 ratio) from the ethyl-acetate crude extract at a stock concentration of $1.398 \mathrm{mg} / \mathrm{ml}$. The compounds, 7-methoxy-apidenindin and luteolinidin accounted for 3.04\% and $52.76 \%$, respectively, of the total count ion content in the crude extract. 


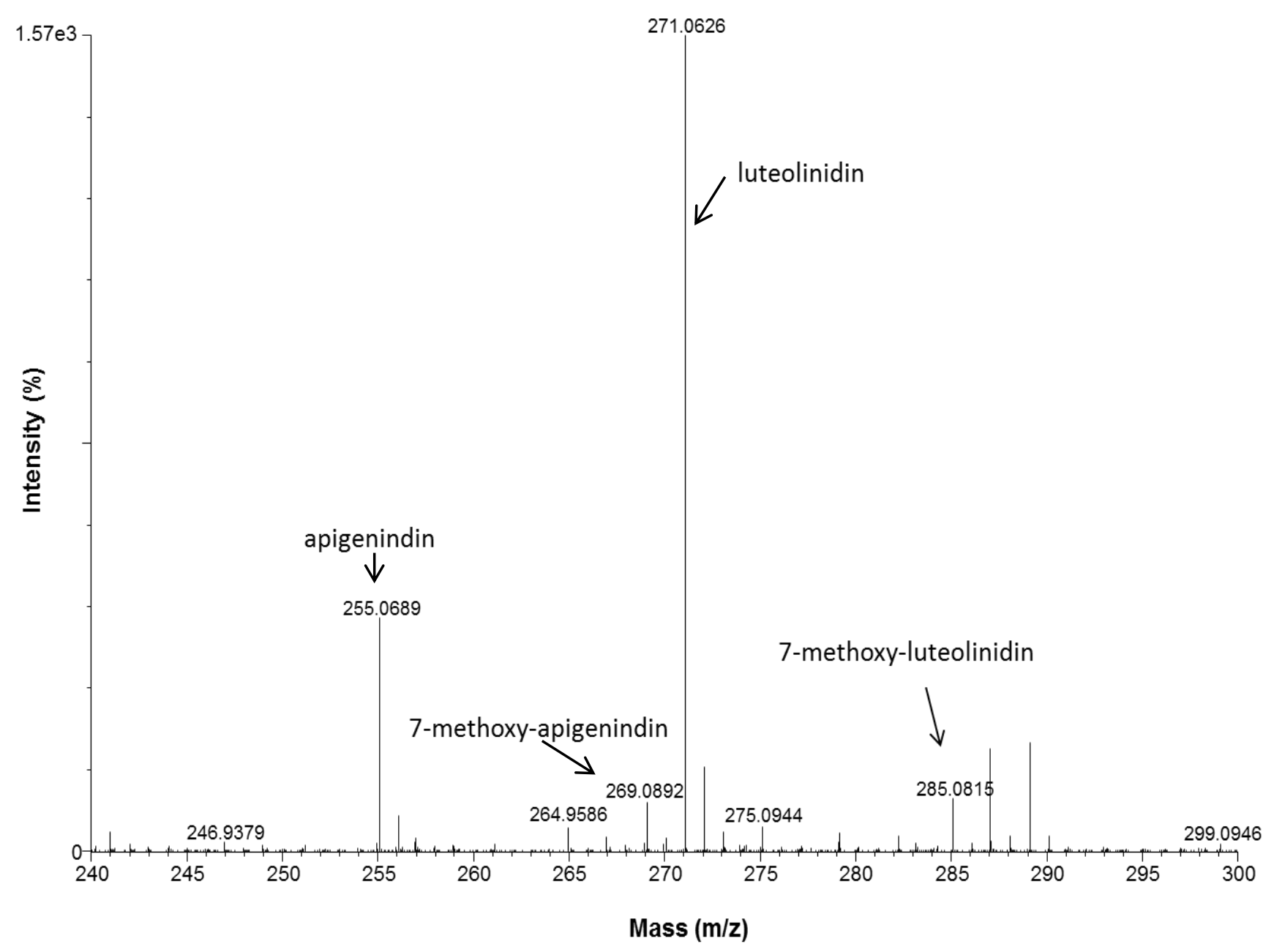

Figure 3. ESI-MS chromatogram of compounds extracted from S. bicolor. The 3-deoxyathocyaninidins were fractionated in chloroform from the ethyl-acetate crude extract at a stock concentration of $3.80 \mathrm{mg} / \mathrm{ml}$. Apigeninindin, 7-methoxy-apigenidin, luteolinidin and 7-methoxy-luteolinidin accounted for $4.61 \%, 2.75 \%$, $36.87 \%$ and $2.50 \%$, respectively, of the total ion count in the crude extract. 


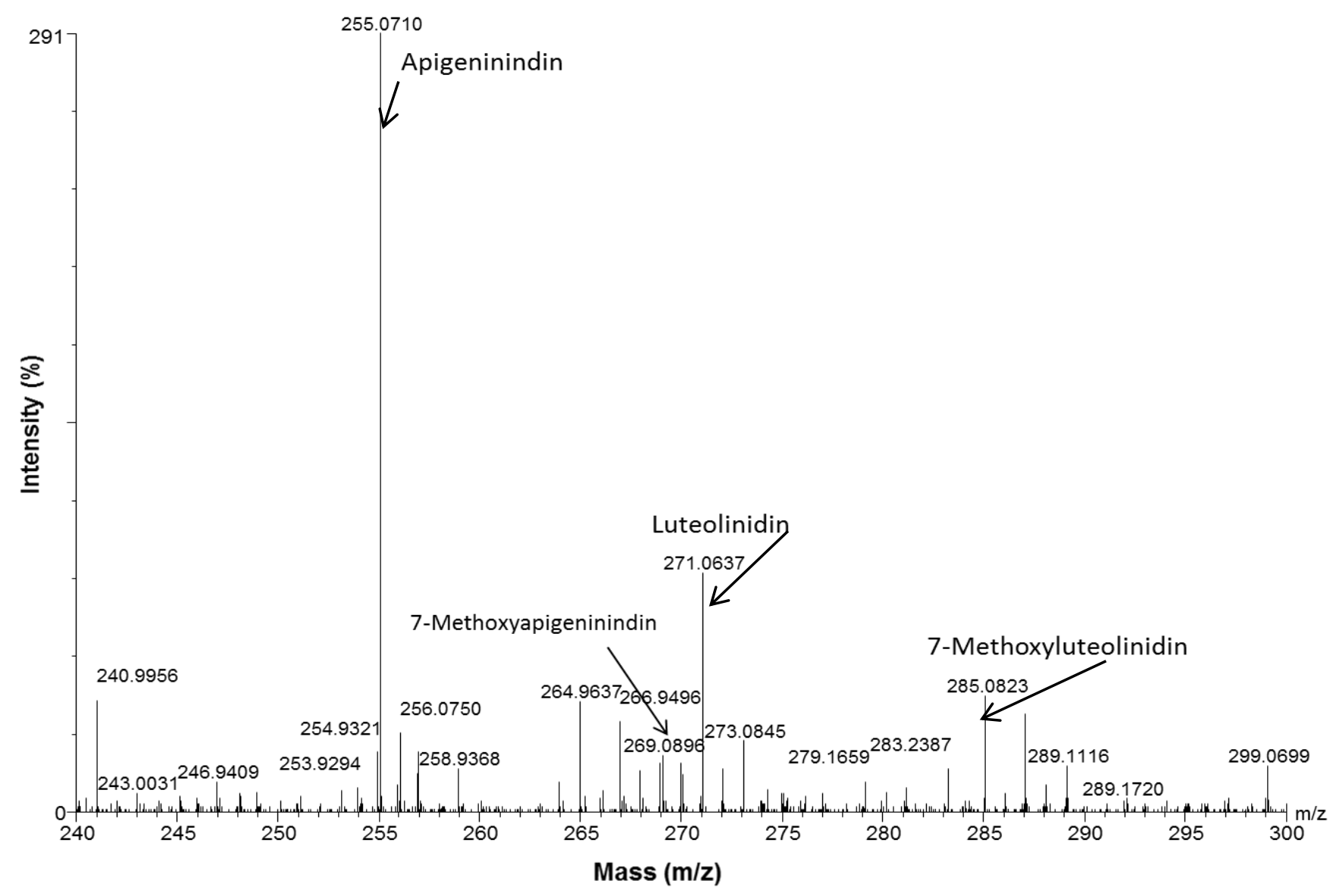

Figure 4. ESI-MS chromatogram of compounds extracted from S. bicolor. The 3-deoxyathocyaninidins were fractionated in hexane-methanol from the ethyl-acetate crude extract at a stock concentration of $18.389 \mathrm{mg} / \mathrm{ml}$. Apigeninindin, 7-methoxy-apigenidin, luteolinidin and 7-methoxy-luteolinidin accounted for 4.44\%, 0.72\%, $1.38 \%$ and $0.57 \%$, respectively, of the total ion count in the crude extract. 
A.

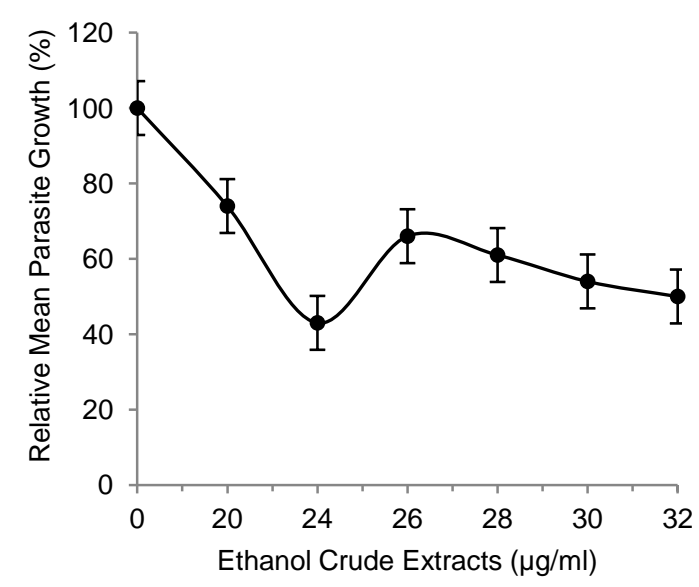

c.

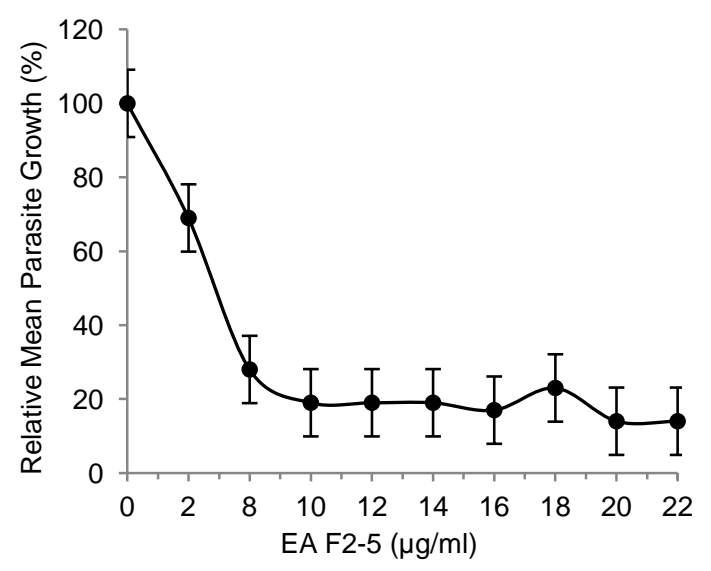

B.

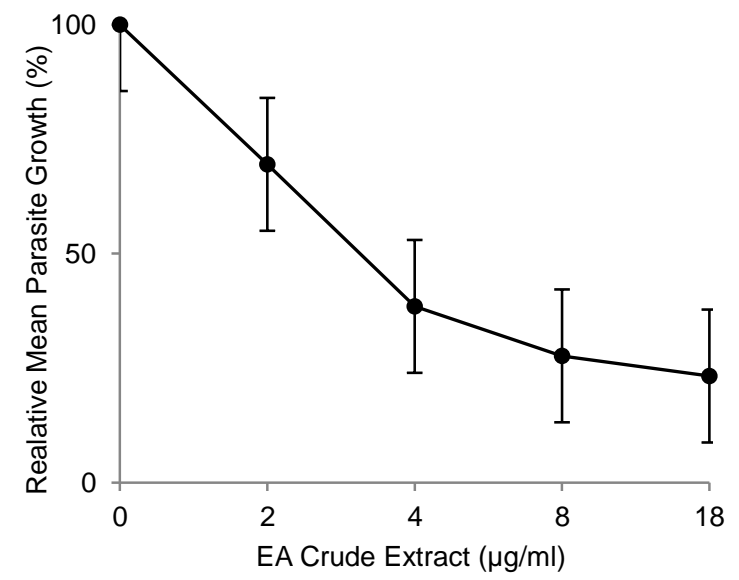

D.

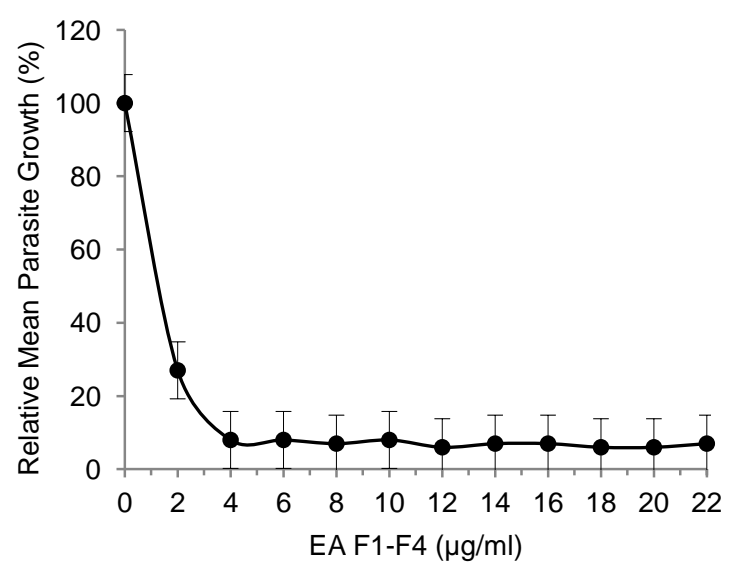

Figure 5. Growth curves for $T$.gondii tachyzoites in cultures treated with varying concentrations of compounds. Confluent human foreskin fibroblasts (HFF) were inoculated with $T$. gondii tachyzoites and immediately treated with varying concentrations $(\mu \mathrm{g} / \mathrm{ml})$ of (A.) Ethanol crude extract, (B.) Ethyl acetate crude extract, (C.) Ethyl acetate fraction F2-5 and (D.) Ethyl acetate fraction F1-F4, for 48 hours. The graphs depict compound dose-response patterns of decline in growth.. The data are representative of means of three independent assays at 95\% confidence limit (95\% CL). 
A.

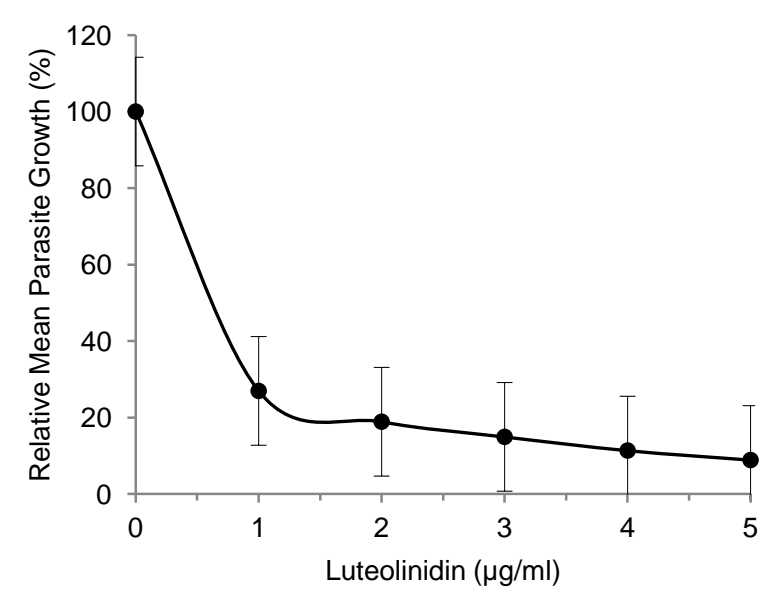

c.

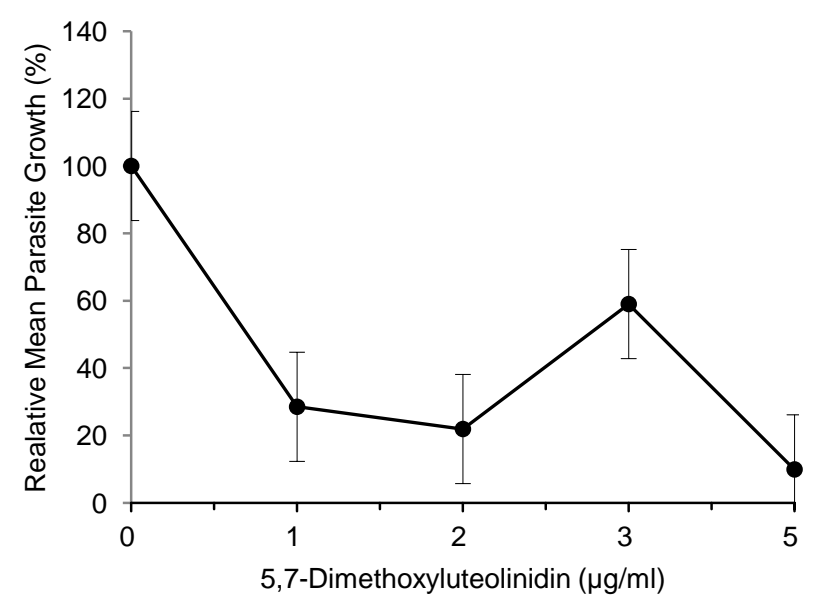

B.

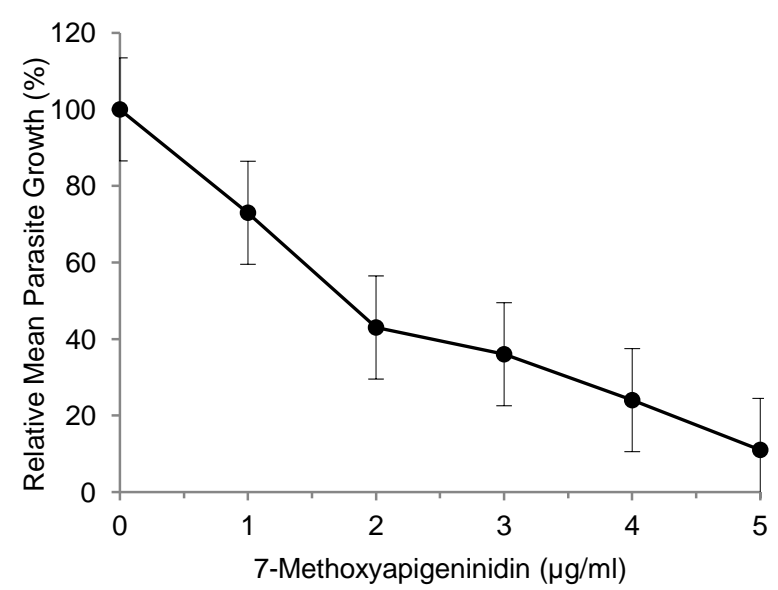

D.

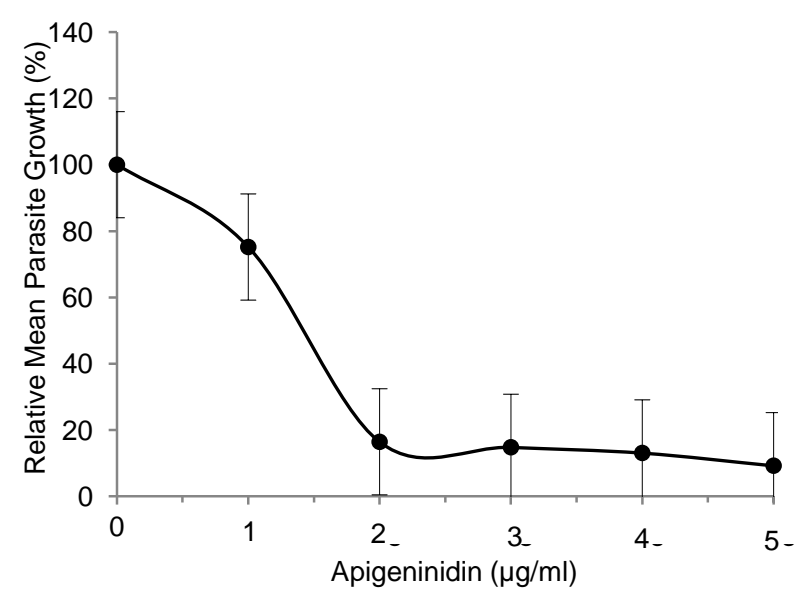

Figure 6. Growth curves for $T$.gondii tachyziotes in cultures treated with varying concentrations of pure compounds. Confluent human foreskin fibroblasts (HFF) were inoculated with T. gondii tachyzoites and immediately treated with varying concentrations $(\mu \mathrm{g} / \mathrm{ml}$ ) of (A.) Luteolinidin, (B.) 7-Methoxyapigeninidin, (C.) 5,7-

Dimethoxyluteolinidin and (D.) Apigeninidin, for 48 hours. The graphs depict compound dose-response decline in growth.. The data are representative of means of three independent assays at 95\% confidence limit (95\% CL). 